\title{
Energy Enhanced Base Station Controlled Dynamic Clustering Protocol for Wireless Sensor Networks
}

\author{
K. Padmanabhan and P. Kamalakkannan
}

\begin{abstract}
The energy efficiency is the determinant aspect of the wireless sensor network life time. Various routing protocols are used to reduce the energy consumption and to improve the network lifetime. To support high scalability and better data aggregation, sensor nodes are often grouped into disjoint, non overlapping subsets called clusters. Clusters create hierarchical WSNs which incorporate efficient utilization of limited resources of sensor nodes and thus extends network lifetime. This paper discusses an improved clustering protocol to minimize the energy consumption and maximize the network life-time. The simulation results show that the proposed algorithm reduces the overall energy consumption and improves the lifetime over its comparatives.
\end{abstract}

Index Terms-Clustering, EEBDCP, energy enhancement, LEACH, residual energy, wireless sensor networks.

\section{INTRODUCTION}

Wireless sensor networks consist of thousands of low-power sensor nodes. They are normally deployed in an unattended environment. Sensor nodes have limited sensing and computational capability. Recent developments in this field have made the sensor nodes small in size and low in cost. The sensor nodes are used in different applications such as military and security, environmental monitoring, automobile industries, patient health monitoring, constructions, and many other applications [1]. Sensor nodes consist of sensing unit, processing unit, transceiver unit, and power unit. The sensing unit consists of sensor and an ADC. The sensor node senses the environment and the $\mathrm{AD}$ converter converts the sensed analog data to digital and transmits the same to the processing unit. The processing unit consists of processor and storage. The processor performs the data aggregation and the aggregated data is transmitted to the transceiver unit. The transceiver unit consists of transmitter radio and receiver radio. The transmitter radio transmits the aggregated data to the sink. The power unit consists of low power batteries. Usually the batteries cannot be replaced or rechargeable [2]. A sensor network life-time is normally expected to last few months to one year without recharging. The energy consumption can be reduced by making some sensors to sleep [3]. The researches show that transmission and reception of data consumes energy in the sensor network. The nodes in the sleeping mode are restricted to

Manuscript received October 5, 2012; revised December 6, 2012.

K. Padmanabhan is with Mahendra College of Engineering, Salem, and Tamilnadu, India (e-mail: padma_india75@yahoo.co.in).

P. Kamalakkannan is with the Department of Computer Science, Govt. Arts College (Autonomous), Salem, Tamilnadu, India (e-mail: kamal_karthi96@yahoo.co.in).. consume the energy.

The sensors can transmit the data to the sink either directly or through the intermediate nodes. In the direct transmission, the node which is far away from the sink has to spend more energy than the node which is nearest to the sink. In multi-hop routing protocol, the data is transmitted to the sink through the intermediate nodes. In this, the node which is nearest to the sink will drain out its energy very quickly [4]. Various routing protocols have been proposed to improve the network life-time. Data centric approach is one of the approaches that uses attribute base addressing to perform the collective sensing. In this routing, sensor nodes are assigned tasks based on interest disseminations that originate from another node in the network [1]. In this paper, we focus on the energy efficient clustering algorithm for wireless sensor network. We propose a modified algorithm of BCDCP [5] called "Energy efficient base station controlled dynamic clustering Protocol". Our proposed protocol facilitates the nodes with more residual energy have more chances to be selected as cluster head. In order to extend the lifetime of the whole sensor network, energy load must be evenly distributed among all sensor nodes so $t$ hat the energy at a single sensor node or a small set of sensor nodes will not be drained out very soon.

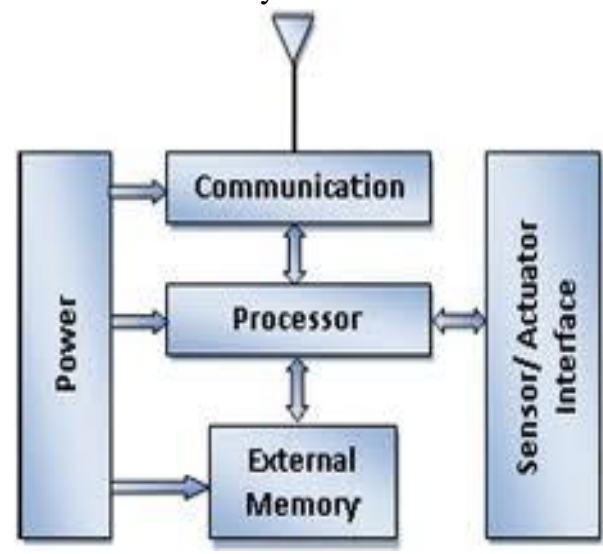

Fig. 1. Wireless Sensor node architecture

In Section on II related works for our protocol is described. Section III describes the proposed system model. Simulation results are shown in Section IV. Finally, we provide concluding remarks in Section V.

\section{RELATED WORKS}

The clustering technique is used reduce the energy consumption. In the traditional clustering approach, the cluster nodes select their head on random basis. The cluster-head selection is static. The node which is selected 
as cluster-head will serve as head during the entire network lifetime. Since the senor nodes have limited energy, the cluster-head node will drain out its energy quickly. The death of a node in the sensor network indicates the end of the network life-time. The death of a node will make the network ineffective. In the classical clustering approach, the LEACH (Low Energy Adaptive Clustering Hierarchy sensor nodes elect their head randomly. To improve the network lifetime, the new cluster-head will be selected for every round [4]. The operations of $\mathrm{LEACH}$ are divided into rounds. Each round consists of setup phase and steady-state phase. In the setup phase, the cluster-head is selected based on the threshold value

$$
T(n)=\frac{p}{1-p \times\left(r \bmod \frac{1}{p}\right)}, n \in G, \quad 0 \text { otherwise }
$$

where $p$ is the desired percentage of cluster head, $r$ is the current round, and $G$ is the set of nodes that have not been cluster heads in the last $1 / p$ rounds. All the nodes in the cluster will have a chance to become a cluster head. The elected $\mathrm{CH}$ will broadcast an advertisement message using a non-persistent CSMA (Carrier Sense Multiple Access) MAC protocol [6] to the member nodes about its status. This small message contains the node Id and a header. The member nodes determine their cluster based on the received signal strength. Then they transmit a join request to the $\mathrm{CH}$ using a non-persistent CSMA MAC protocol. This short message consisting of node's ID and cluster head's ID. The CH sets up a TDMA schedule and transmits it to the member nodes. The member nodes will be in sleeping mode except during their transmission time. In the steady-state phase, the member nodes send their data to the $\mathrm{CH}$ during their TDMA slot. The slot is constant, so the time to send the data depends on the number of nodes in the cluster.

A centralized version of $\mathrm{LEACH}, \mathrm{LEACH}-\mathrm{C}$, is proposed in [2]. LEACH-C utilizes the base station for cluster formation. During the setup phase of $\mathrm{LEACH}-\mathrm{C}$, the base station receives information regarding the location and energy level of each node in the network. Using this information, the base station finds a predetermined number of cluster heads and configures the network into clusters. The cluster groupings are chosen to minimize the energy required for non-cluster- head nodes to transmit their data to their respective cluster heads. Although the other operations of LEACH-C are identical to those of $\mathrm{LEACH}$, results presented indicate a definite improvement over LEACH. In BCDCP, the base station changes cluster heads randomly, composes cluster-to-cluster routing paths, and achieves other energy intensive works such as data aggregation, compression, and fusion [5]. The key ideas in BCDCP are the formation of balanced clusters where each cluster head serves an approximately equal number of member nodes to avoid cluster head overload, uniform placement of cluster heads throughout the whole sensor field, and utilization of cluster head to cluster head $(\mathrm{CH}-$ to- $\mathrm{CH})$ routing to transfer the data to the base station. As LEACH-C, in the setup phase, energy information that sent by all sensor nodes is used to compose clusters. The base station uses balanced clustering technique [7] for distribution of the load of cluster heads, and iterative cluster splitting algorithm to get ideal number of clusters. After that, it makes a multiple $\mathrm{CH}$-to- $\mathrm{CH}$ routing paths $[8,9]$. Finally, it creates schedule for each cluster and broadcasts it to sensor networks. In the data communication phase, cluster heads transfer data from sensor nodes to the base station according to the $\mathrm{CH}$-to- $\mathrm{CH}$ routing paths after processing it. In CBCDACP (Central Base station Controlled Density Aware Clustering Protocol), an optimum set of cluster heads is selected by the centralized clustering algorithm. The nodes having higher sensor density are selected as cluster heads and incur minimum cost. In the setup phase, the cluster head candidates are selected from the set of alive nodes whose energy is higher than the average energy in the current round. For $\mathrm{CH}$ selection, nodes are chosen from the candidate nodes depending on which have higher density. A node will have higher density if it has more neighbor nodes at closest distance than others [10]. Energy efficient Multi-Hierarchy Clustering protocol (EMHC) has seven phases [12]. In the cluster head selection phase, the residual energy and coverage are used as parameters for cluster head selection. Two levels of cluster heads are selected. The sensed data are transmitted to the base station through the multi-level cluster heads.

EEBDCP is a modified cluster-based routing protocol, which utilizes a high-energy base station to set up clusters and routing paths, perform randomized rotation of cluster heads, and carry out other energy-intensive tasks. The key ideas in EEBDCP are the formation of balanced clusters where each cluster head serves an approximately equal number of member nodes to avoid cluster head overload, uniform placement of cluster heads throughout the whole sensor field, and utilization of cluster-head-to-cluster head $(\mathrm{CH}-$ to- $\mathrm{CH})$ routing to transfer the data to the base station. EEBDCP yields an improved system lifetime and better energy savings over the above mentioned clusterbased routing protocols.

\section{EEBDCP: ENERGY ENHANCED BASE STATION CONTROLLED DYNAMIC CLUSTERING PROTOCOL}

We propose a modified Energy enhanced dynamic clustering protocol (EEBDCP) for wireless sensor networks. This routing protocol provides balanced energy consumption and maximum network life time. This protocol selects a cluster head based on residual energy of the sensor nodes. The cluster heads are selected by the base station. The minimum spanning tree algorithm is used to connect the cluster heads. All member nodes in the clusters send their data to the CHs. Then the $\mathrm{CHs}$ select on $\mathrm{CH}$ leader to collect the data from the other $\mathrm{CHs}$ and finally transmit the collected data to the Base station. The $\mathrm{CH}$ leader node is selected dynamically for each round.

\section{A. The System Model and Assumptions.}

- The proposed protocol assumes the following properties.

- The sensor nodes are energy constrained with a uniform initial energy allocation.

- The sink located very far from the cluster-heads.

- All the sensor nodes are stationary with limited energy. 
- All the sensor nodes are equipped with power control capabilities to vary their transmitting power.

- The network is assumed to be continuous data delivery model.

\section{B. Radio Model}

A radio communication subsystem consists of transmitter, receiver, antennae, and an amplifier. In this analysis, we use the same radio model used in [2].

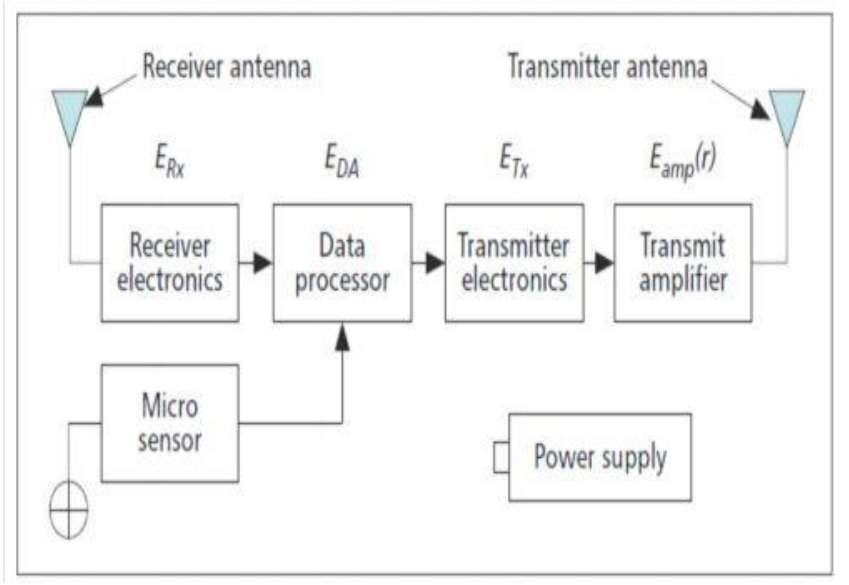

Fig. 2. Radio model

Transmit and receive energy for the transfer of a k-bit message is given by equation 1 and 2 respectively.

$$
\begin{gathered}
E_{T}(k, r)=E_{T x} k+\mathrm{E}_{\mathrm{amp}}(r) k \\
E_{R}(k)=E_{R x} k(2)
\end{gathered}
$$

Equation (1) denotes the total energy dissipated in the transmitter of the source node, and equation 2 represents the energy incurred in the receiver of the destination node. The parameters $E_{T x}$ and $E_{R x}$ are per bit energy dissipations for transmission and reception, respectively. $E_{a m p}(r)$ is the energy required by the transmit amplifier to maintain an acceptable signal-to-noise ration in order to transfer data messages reliably. As is the case in [2], we use both the free space propagation model and the two-ray ground propagation model to approximate the path loss sustained due to wireless channel transmission.

Given threshold transmission distance of $r 0$, the free space model is employed when $r \leq r 0$, and the two-ray model is applied for cases where $r \geq r 0$. Using these two models, the energy required by the transmit amplifier $E_{a m p}(r)$ is given by

$$
\begin{gathered}
E_{a m p}(r)={ }_{F S} r^{4}, \quad r \leq r 0 \\
T R r, \quad r>r 0
\end{gathered}
$$

where $F S$ and $T R$ denote transmit amplifier parameters corresponding to the free space and two-ray models, respectively, and r0 is the threshold distance given by

$$
R_{0}=\sqrt{ } F S / T R
$$

We assumed the same parameters used in [2] for all experiments. $E_{T x}=E_{R x}=50 \mathrm{~nJ} / \mathrm{bit}, \quad F S=10 \mathrm{pJ} / \mathrm{bit} / \mathrm{m} 2$, and $\mathrm{TR}=0.0013 \mathrm{pJ} / \mathrm{bit} / \mathrm{m} 4$. The energy cost of data aggregation is set as $E_{D A}=5 \mathrm{~nJ} / \mathrm{bit} / \mathrm{message}$.

\section{Cluster Construction Phase}

In the cluster construction phase, the iterative cluster splitting algorithm is applied to find the number of clusters and CHs. This algorithm splits the cluster into two subclusters. This process will be continuing by splitting the sub-clusters into smaller clusters. This algorithm ensures that the selected $\mathrm{CHs}$ are uniformly placed throughout the sensor field by maximizing the distance between $\mathrm{CHs}$ in each splitting step. The balanced clustering technique is used to make the clusters equal in size [7]. The minimum spanning tree algorithm is applied to connect the $\mathrm{CHs}$ to form the shortest path between them [12].

\section{Algorithm 1: Cluster construction}

1: Input: Residual energy at each node

2: Output: Balanced clusters and $\mathrm{CHs}$

3: Initialization: compute the average residual energy of all nodes;

4: Select the set of non-sensing nodes $S$ whose residual energy is higher than the average;

5: Repeat

6: Select as cluster heads the two nodes in $\mathrm{S}$ with maximum separation distance between them;

7: Assign all other nodes to their closest cluster head, leading to formation of two groups;

8: Balance the two groups, using algorithm 2;

9: Split S into two sets, S1 and S2 whose elements are the group members in step 8;

10: Until $\mathrm{N}$ clusters have been selected

In the initial round, the sensor nodes randomly choosing one $\mathrm{CH}$ and forward the energy and location information to base station. Then the base station computes the average energy level of all the nodes. A set of nodes which are having energy level greater than the average energy level will be selected by the base station.

\section{Algorithm 2: Balanced cluster}

1: Input: $M, N$, and current size of the clusters to be balanced

2: Output: balanced clusters

3: Initialization: assign the current size of the clusters to vector $\mathrm{A}=\{A i\}$, where $A i=$ size of cluster $I$;

4: Compare current cluster sizes with the desired size:

$\mathrm{A}=\mathrm{A}-\mathrm{M} / \mathrm{N}$;

5: For each element $A_{4} i$ of $A$ do

6: While $A i \neq 0$ do

7: If $A i>0$ then

8: $\quad$ Select the node $\mathrm{z}$ in cluster $\mathrm{i}$ with longest distance to its $\mathrm{CH}$;

9: Select the closest $\mathrm{CH} j(j \neq i)$ to node $\mathrm{z}$ such that $A j<0$;

10: Decrement size of cluster $i, A i=A i-1$;

11: Increment the size of cluster $j, A j=A j+1$;

12: Elsif $A i<0$ then

13: Select the node $\mathrm{z}$ in cluster $\mathrm{j}$ with shortest distance to cluster head i such that $A j>0$;

14: Assign $z$ from cluster $j$ to cluster $i$; 
15: Increment the size of cluster $i, A i=A i+1$;

16: Decrement the size of cluster $j, A j=A j-1$;

17: End if

18: End while

19: End for

$\mathrm{CHs}$ for the current round will be selected from the set of nodes. The node with the highest energy is selected as $\mathrm{CH}$. The base station broadcast the $\mathrm{CH}$ selection information and the TDMA transmission schedule to the member nodes. The energy consumption task is performed by the base station.

\section{Data Communication Phase}

In the data communication phase, each sensor node senses the field and transmits the gathered data to the corresponding CHs. The member nodes use the TDMA schedule to transmit the data to the $\mathrm{CH}$. The remaining nodes turn off their radio and they will be in sleeping mode. The CDMA spreading code is used to avoid the interference between the clusters. Each cluster will be using a separate spread code for its transmission. The $\mathrm{CHs}$ aggregate the received data and transfers it to the $\mathrm{BS}$ through the $\mathrm{CH}$ Leader node. The $\mathrm{CH}$ node which is having the highest residual energy will be selected as the $\mathrm{CH}$ leader node and all other $\mathrm{CHs}$ transfers the aggregated data to the base station through this $\mathrm{CH}$ leader node.

\section{PERFORMANCE ANALYSIS}

To assess the performance of EEBDCP, we simulated EEBDCP performance using Network Simulator2 and compared its performance with other clustering-based routing protocols such as LEACH and EMHC. Performance is measured by average energy dissipation, system life-time, total messages delivered successfully, base station location, the number of nodes that are alive and the throughput. We have used 200 nodes where each node is assigned an initial energy of $2 \mathrm{~J}$. The number of data frames transmitted for each round is set at 50; the data message size for all simulations is fixed at 500 bytes, of which 25 bytes represent the length of the packet header.

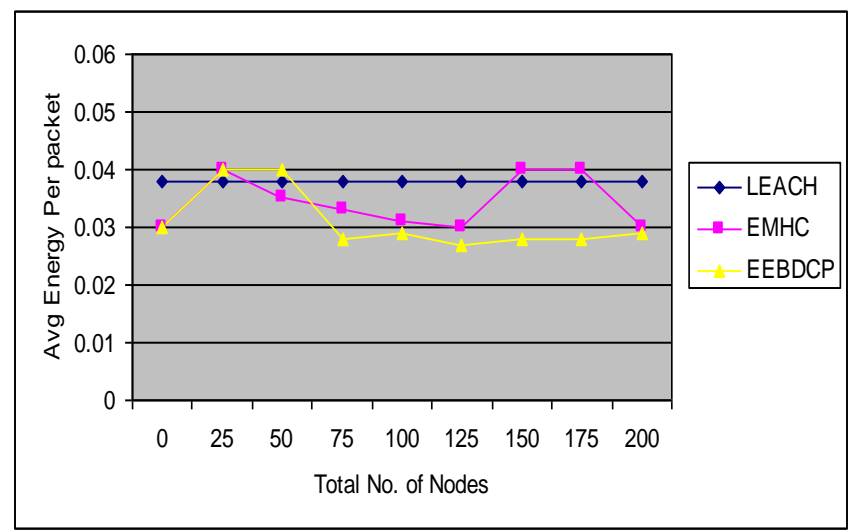

Fig. 3. Average energy dissemination per packet

Fig. 3 shows a comparison of average energy dissipation over the number of nodes. EEBDCP considerably reduces the energy consumption compared to EMHC and LEACH. The EEBDCP uses minimum energy to transmit the packets. It also uses single hop transmission to the CHs. The average energy dissipation is $15 \%$ less than EMHC. The EEBDCP consumes less energy than the EMHC. It shows that EEBDCP performs better than EMHC in terms of energy efficiency as well as network life time.

Fig. 4 shows a comparison of throughput of the protocol. The total number of packets sent per second is analyzed in this figure. EEBDCP sent more packets to the sink than the EMHC. It outperforms the comparatives based on the throughput.

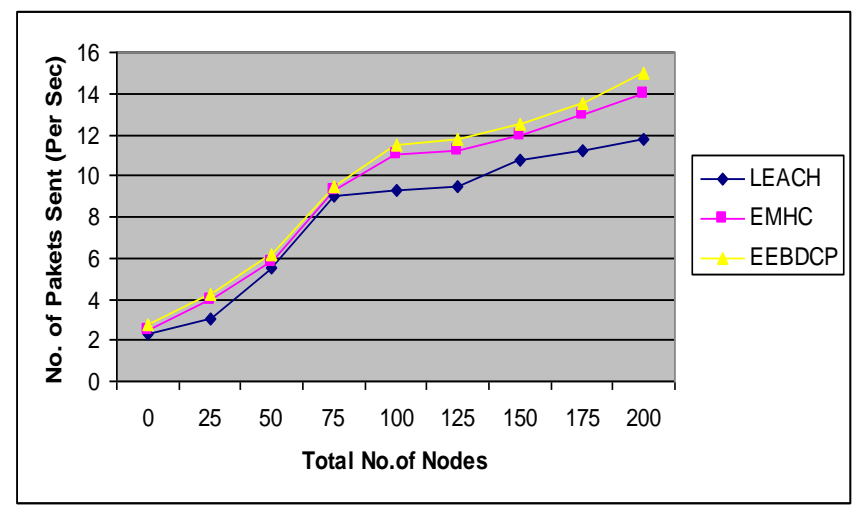

Fig. 4. Throughput on the no. of packets

Fig. 5 shows the impact that the distance to the base station on the network lifetime. The number of rounds in EEBDCP is 1800 when the base station is $150 \mathrm{~m}$ distance from the nearest node while in EMHC, the number of rounds is 1550 . The simulation shows that EEBDCP outperforms EMHC when the base station is far from the network.

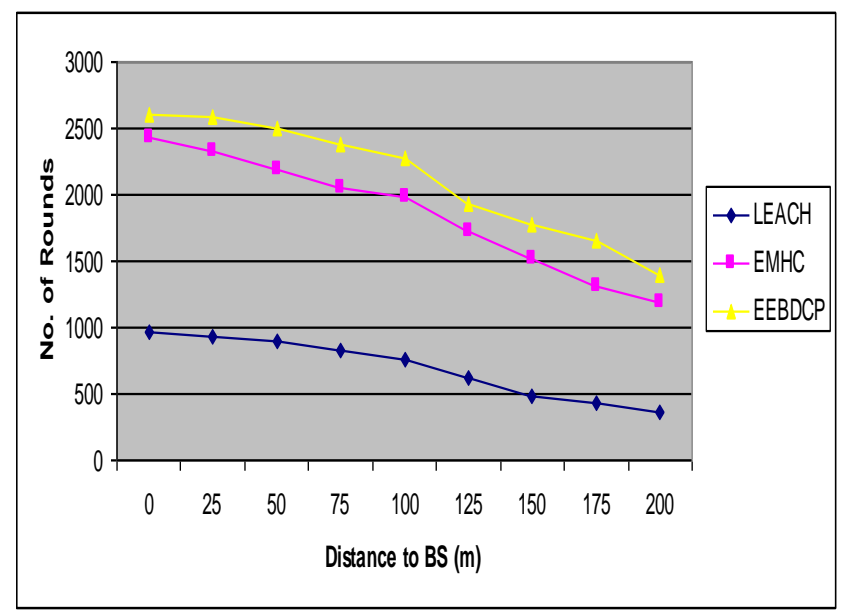

Fig. 5. Network life-time under different BS locations

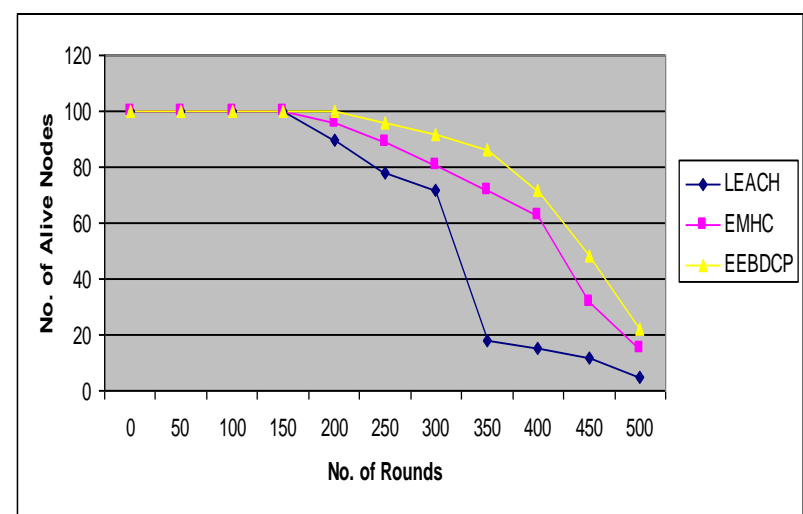

Fig. 6. Comparison of Total no. of living nodes in each round

Fig. 6 shows the number of living nodes in EEBDCP and EMHC. The nodes start to die after 160 rounds in EMHC. In EEBDCP, the nodes start to die after 210 rounds. It shows 
that the number of living nodes is $25 \%$ greater than the number of living nodes in EMHC. This simulation clearly shows that the network life is increased in case of EEBDCP.

\section{CONCLUSION}

In this paper, we proposed a clustering protocol, EEBDCP that utilizes the services of high-energy base station to perform most energy-intensive tasks. By using the base station, the sensor nodes are relieved of performing cluster setup, cluster head selection, routing path formation. Performance of the proposed EEBDCP protocol is assessed by simulation and compared to clustering-based protocol EMHC. The simulation results show that EEBDCP outperforms its comparatives by uniformly placing cluster heads throughout the whole sensor field, performing balanced clustering, and using a $\mathrm{CH}$-to- $\mathrm{CH}$ routing scheme to transfer fused data to the base station. It is also observed that the performance gain of EEBDCP over its counterparts increases with the area of the sensor field. Therefore, it is concluded that EEBDCP provides an energy efficient routing scheme suitable for a vast range of sensing applications.

\section{REFERENCES}

[1] I. F. Akiyildiz and W. Zu, "A survey on sensor networks," IEEE Communications magazine, August, 2002

[2] W. B. Heinzelman, A. P. Chandrakasan, and H. Balakrishnan, "An application specific protocol architecture for wireless microsensor networks," IEEE communications, 2002.

[3] L. Wang and Y. Xiao, "A survey of energy efficient scheduling mechanisms in sensor networks," Springer, May 2006

[4] W. R. Heinzelman, A. P. Chandrakasan, and H. Balakrishnan, "Energy efficient communication protocol for wireless micro sensor networks," IEEE transactions on wireless communications, vol.1, no. 4 , Oct 2002

[5] S. D. Muruganathan, D. C. F. Ma, R. I. Bhasin, and A. O. Fapojuwo, "A Centralized Energy-Efficient Routing Protocol for Wireless Sensor Networks," IEEE Radio communications, 2005
[6] K. Pahlavan and A. Levesque, Wireless information networks, Newyork: Wiley 1995

[7] S. Ghiasi et al., "Optimal energy aware clustering in sensor networks," MDPI sensors, vol. 2, no. 7, pp. 258-69, July 2002

[8] C. Li, H. Zhang, B. Hao, and J. Li, "A Survey on Routing Protocols for Large-Scale Wireless Sensor Networks," Sensors, vol. 11, pp. 3498-3526, 2011.

[9] O. Buyanjargal and Y. Kwon, "Adaptive and Energy Efficient Clustering Algorithm for Event-Driven Application in Wireless Sensor Networks," Journal of Networks, vol. 5, no. 8, august 2010.

[10] J. Ferdous, J. Ferdous, and T. Dey, "A Comprehensive Analysis of CBCDACP in Wireless Sensor Networks," Journal of communications, vol. 5, no. 8, august 2010

[11] H. Shen, "Finding the k-most vital edges with respect to minimum spanning tree," in Proc. National Aerospace and Electrical Conference, vol.1, July 1997, pp. 255-62.

[12] B. Nazir and H. hasbullah, "Energy efficient multi-hierarchy clustering protocol for wireless sensor networks," in Proc. ICIIT, 2010.

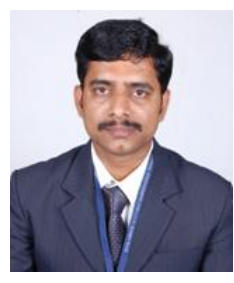

K. Padmanabhan was born at Salem in the year 1975. He received M.C.A degree in 1998from the University of Madras in India. He is working as an Asst.Professor in the department of computer applications at Mahendra College of Engineering, Salem, India. He is pursuing Ph.D degree in the area of energy conservation. His research interest includes Wireless sensor networks and Wireless Adhoc Networks.

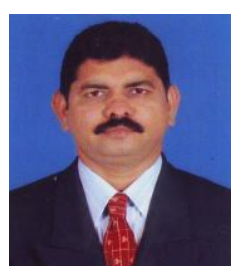

P. Kamalakkannan was born at Tiruchengode in the year 1967. He received his B.Sc and M.C.A degrees in 1988 and 1991 from Bharathiar University, India. He has obtained his Ph.D degree in Computer Science in the year 2008. He is working as Asst. Professor in the department of Computer Science, Govt. Arts College (Autonomous), Salem, India. His research interest includes Distributed Systems, Pervasive Computing, and Wireless Adhoc networks, Wireless sensor networks. He has published so many research papers in the national and international journals. Dr. P. Kamalakkannan is a Life member of Computer Society of India and Indian Society for Technical Education. 